\title{
Inclusión educativa. Actitudes y estrategias del profesorado
}

\section{Inclusive education. Attitudes and strategies of teachers}

\begin{abstract}
Resumen
La educación inclusiva da respuesta al alumnado escolarizado en los centros educativos, independientemente de sus características y necesidades. Esto ha supuesto cambios metodológicos, curriculares y actitudinales por parte del profesorado.
\end{abstract}

Los objetivos del estudio son evaluar las actitudes de los docentes hacia la inclusión, conocer las estrategias utilizadas en el aula y evaluar la incidencia de variables sociodemográficas y relacionadas con la práctica profesional, utilizando una metodología cuantitativa a través del cuestionario Actitudes y prácticas del profesorado relativas a la inclusión.

La muestra está formada por 82 docentes. Los resultados determinan actitudes positivas hacia la inclusión y un uso frecuente de estrategias inclusivas en las aulas, a mayor actitud positiva mayor uso de estrategias. Las variables profesionales tienen mayor incidencia que las sociodemográficas tanto en las actitudes como en las estrategias utilizadas por los docentes.

\section{Palabras clave}

Inclusión educativa, actitudes, estrategias docentes, necesidades educativas especiales, discapacidad, metodología cuantitativa.

\author{
Abstract \\ Inclusive education serves the \\ entire population of students at \\ schools independent of their diverse \\ characteristics and needs. This \\ situation has required methodological, \\ curricular and attitudinal changes \\ among teachers.
}

The objectives of this study are to evaluate teachers' attitudes towards inclusion and the strategies used in the classroom. Furthermore this study pretends to evaluate the influence of sociodemographic variables and variables related to professional practice on these attitudes and strategies. A quantitative methodology, which included the application of the questionnaire Attitudes and practices of teachers related to inclusion, was used.

The sample is formed by 82 teachers. The results indicate the presence of positive attitudes towards inclusion and a frequent use of inclusive strategies. There is a correlation between the attitudes and the use of inclusive strategies: the more positive the attitudes the more use of inclusive strategies. Professional variables have a greater impact on the attitudes and the strategies than sociodemographic variables.

Keywords:

Inclusive education, attitudes, strategies teachers, special educational needs, disability, quantitative methodology.

\author{
Paula Garzón Castro \\ <paulagc@usal.es> \\ Universidad de Salamanca
}

\section{Ma Isabel Calvo Álvarez} <isabelc@usal.es>

Universidad de Salamanca

Ma Begoña Orgaz Baz

<borgaz@usal.es>

Universidad de Salamanca

Para citar:

Garzón, P. et al. (2016): "Inclusión educativa. Actitudes y estrategias del profesorado". Revista Española de Discapacidad, 4 (2): 25-45.

Doi: <https://doi.org/IO. 5569/23405 I04.04.02.02>

Fecha de recepción: I6-I 2-20I 5 Fecha de aceptación: 27-09-20I6 


\section{Introducción}

La educación inclusiva es el resultado de un largo camino de transformación de la educación. Los modelos inclusivos empezaron a cobrar relevancia en I994, cuando la Declaración de Salamanca proclama la inclusión como el medio más eficaz para educar a todos los alumnos en el sistema educativo ordinario y que éstos accedan a una educación de calidad independientemente de sus características y necesidades individuales (Unesco, I994). La inclusión como principio rector de la educación ha sido fundamental para convertir las escuelas en centros inclusivos que den respuesta a la diversidad del alumnado, no únicamente al alumnado con necesidades educativas especiales (Ainscow, 20I2). El modelo de educación actual basado en la equidad, la participación y la efectividad, principios todos compartidos a nivel internacional, debe garantizar el desarrollo de las capacidades del alumnado, adaptarse a la diversidad de necesidades indistintamente de sus condiciones personales, sociales y culturales y trabajar por y para una educación inclusiva; es un derecho de todos acceder a una educación de calidad.

Los pasos que se están generando hacia la educación inclusiva no están resultando sencillos, el camino es largo y serpenteante. Actualmente, en este viaje se reconoce que en el éxito o fracaso de modelos inclusivos en los centros influyen distintos factores, uno de ellos son las actitudes del profesorado hacia la inclusión de personas con discapacidad en las aulas ordinarias (Avramidis y Norwich, 2002; Dart, 2007; Scruggs y Mastropieri, 2003) y otro el desarrollo de estrategias educativas inclusivas que implementa el profesorado en las que participan todos los alumnos (Chiner, 20II). Ambos aspectos han sido objeto de estudio de muchas investigaciones con el fin de conocer las actitudes del profesorado hacia la discapacidad para modificar y/o cambiar las actitudes negativas en actitudes positivas y conseguir la inclusión de todos los alumnos (Dendra et al., I991; García y Alonso, I985;
Verdugo et al., I994). Conscientes de que un profesor del siglo XXI debe ser un profesor no solo capaz de reflexionar sobre su práctica sino capaz de adquirir actitudes positivas hacia la diversidad se planteó este trabajo. Las actitudes que manifiestan los docentes en las aulas pueden convertirse en una barrera o un facilitador en el proceso de enseñanza-aprendizaje de todos los alumnos a la vez que repercuten en la utilización de un mayor o menor uso de estrategias inclusivas.

Como se ha señalado anteriormente, en el sistema educativo se han ido produciendo de forma gradual cambios que determinan y muestran el camino hacia la inclusión educativa de todo el alumnado en el contexto escolar. Es importante contextualizar la inclusión para conocer de dónde venimos y hacia dónde nos dirigimos (Ainscow y Booth, 2000; Granada et al., 2013). Se ha evolucionado de un modelo segregado basado en los déficits a un modelo médico, organicista y biológico con una concepción estática de las diferencias y luego a un modelo de integración bajo la influencia de las corrientes pedagógicas surgidas en Canadá, Suecia y Estados Unidos emergente a partir de los años 70 y paradigma construido bajo el principio de normalización en el que se reconoce el derecho de todas las personas a tener una vida lo más normalizada posible, independientemente de sus características y necesidades, y el derecho a educarse en escuelas ordinarias. En este modelo el alumno se adapta al currículo, a los métodos de enseñanza y a los valores y normas que rigen la escolarización.

El modelo de integración contribuyó a un modelo de inclusión en torno a los años 80 , apoyado por las ideas del Movimiento "Regular Education Initiative" (REI) denominado inclusión (full inclusion). La inclusión educativa aspira a hacer efectivo para todo el alumnado el derecho a una educación de calidad y la igualdad de oportunidades, preocupándose especialmente de aquellos que por diferentes causas están excluidos o en riesgo de marginación. Además, pone el énfasis en que no son los alumnos los que tienen que adaptarse al sistema educativo o al currículum, sino que son éstos los que 
se tienen que adaptarse a las necesidades y características de cada alumno (Abellán et al., 20I0; Arnaiz, 2004; Barrio de la Puente, 2009; Echeita y Ainscow, 20I I; Escudero, 20I 2; Parrilla, 2002; Verdugo, 2003 y 2009), siendo la diversidad fuente de enriquecimiento para toda la comunidad educativa.

Para que se desarrollen modelos educativos inclusivos en los que todos los alumnos estén presentes, participen y aprendan, es necesario repensar la educación, hacer cambios profundos y reestructurar las culturas, las políticas y las prácticas de los centros para dar respuesta a la diversidad y a la heterogeneidad del alumnado (Ainscow et al., 2006).

La inclusión educativa es un proceso complejo que puede llegar a entenderse mejor si se tiene en cuenta que las actitudes de toda la comunidad educativa, en concreto las actitudes del profesorado, son claves para atender la diversidad del alumnado. Las actitudes han sido estudiadas desde el punto de vista psicológico por diferentes autores (Ovejero, I998; Verdugo et al., I994). Para Triandis (I97I: 2-3) una actitud es "una idea cargada de emoción que predispone a una clase de acciones ante un determinado estímulo". En esta definición se reflejan los tres componentes que constituyen una actitud: la idea (componente cognitivo), la emoción asociada a ella (componente afectivo) y la predisposición a la acción (componente conductual) (Verdugo et al., I994). Estudios actuales demuestran cómo estos componentes influyen en la inclusión de alumnos con necesidades educativas especiales. En concordancia con ello, se definen las actitudes como "constructos psicosociales inferidos e individuales que están bajo el dominio de estímulos específicos y objetos de referencia capaces de facilitar una respuesta por parte del sujeto" (Verdugo et al., I995: 83).

Con la finalidad de lograr una educación de calidad para todos en contextos inclusivos, es importante que los docentes tengan actitudes positivas hacia la inclusión y la educación inclusiva, reconozcan la diferencia y la diversidad como valores de la escuela y de la educación y comprendan que el alumnado forma parte del contexto educativo. Si las actitudes son negativas se pueden modificar y/o transformar y desarrollar actitudes más positivas, ¿cómo?, a través de diversos modos de hacer, como el condicionamiento instrumental, el modelado o el refuerzo vicario, la formación e información (Verdugo et al., I994 y I995).

Algunos estudios recogen cómo las actitudes del profesorado dependen en cierta medida de variables como la edad, el género, la formación recibida, los años de experiencia, la etapa educativa, el tipo de centro y el contacto que hayan tenido con personas con discapacidad (Agran et al., 2002; Avramidis et al., 2000; Bhatnagar y Das, 20I4; Cornoldi et al., I998; De Boer et al., 20 I ; Martínez Martín y Bilbao León, 20I I; Mirošević y Lozancić 20I4; Pernell et al., I 985 ; Van Reusen et al., 2000; Stauble, 2009; Vaz et al., 201 5). Otras investigaciones muestran que el tipo de actitud depende de las características de los alumnos, de la disponibilidad de recursos, del apoyo y el tiempo disponible (Cardona, 2006; Cook y Cameron, 20I0; Horne y Timmons, 2009; Huang y Diamond, 2009), así como de la formación docente y capacitación que tienen los maestros para enseñar en clases heterogéneas y diversas (Cardona, 2006; Sánchez et al., 2008).

Todos estos factores influyen no sólo en la actitud del profesorado sino también en las estrategias educativas que utilizan. La forma de enseñar de los docentes en el aula es crucial y va a repercutir en la inclusión de todos los alumnos (Arnáiz, I996; Cook y Friend, I995; Lou et al., 1996). Actualmente, hay una variedad de estrategias que el profesorado puede utilizar en sus clases para dar respuesta a su alumnado partiendo de sus características y capacidades y conseguir su plena inclusión en el aula y en el contexto escolar, como las estrategias organizativas, las estrategias didácticas, la adaptación de actividades, los recursos y los tipos de agrupamientos de los alumnos (Cardona et al., 2000; Chiner, 20I I; Vicuña, 20I3). De esta manera, el alumno tendrá la oportunidad de adquirir los conocimientos y competencias necesarias para desenvolverse 
en su contexto familiar y social y entre todos construir una sociedad inclusiva.

El fin de este trabajo se centra en conocer las actitudes del profesorado y las estrategias que utilizan los docentes de las etapas educativas obligatorias hacia la inclusión de alumnos con necesidades educativas especiales (NEE) derivadas de discapacidad. Como objetivos más específicos se proponen:

I. Evaluar las actitudes de los docentes hacia la inclusión educativa y las estrategias de inclusión que utilizan.

2. Evaluar la incidencia de las variables sociodemográficas y relacionadas con la práctica profesional sobre las actitudes y las estrategias.

3. Analizar si tener una buena actitud hacia la educación inclusiva implica un mayor uso de estrategias inclusivas en las aulas.

\section{Método}

\section{Participantes}

La población objeto de estudio son docentes de Educación Infantil, Primaria y Secundaria que reúnen los siguientes requisitos: imparten docencia en dichas etapas educativas, están en activo y en sus aulas hay alumnos con NEE derivadas de discapacidad.

Para seleccionar a los participantes se emplean técnicas de muestreo no probabilístico, concretamente, el muestreo estratégico o de conveniencia y el muestreo de bola de nieve.

Se contacta con un total de 27 centros educativos: I 5 de Salamanca, 8 de Zamora y 4 de Madrid.

Finalmente, participan 7 centros $(25,9 \%$ de los contactados). En concreto, 3 de Salamanca (20\% de los contactados), 2 de Zamora (25\% de los contactados) y 2 de Madrid ( $50 \%$ de los contactados).
De los 7 centros participantes, 5 son públicos $(33 \%)$, I concertado (9\%) y I privado ( $100 \%$ ). Además, 4 de ellos pertenecen a la zona rural $(57 \%)$ y 3 a contexto urbano (I $5 \%$ ). De todos ellos, 3 centros imparten Educación Infantil y Primaria, 2 Educación Secundaria y Bachillerato, I Educación Infantil, Primaria y ESO y I centro Educación Primaria y Secundaria.

Para disponer de una muestra más amplia, se contacta con un grupo de maestros a través de Facebook. Este grupo permite compartir e intercambiar información, materiales, recursos y recomendaciones, entre otras cosas, relacionadas con la práctica educativa.

Finalmente, la muestra queda constituida por 82 docentes, 53 pertenecen a la plantilla de los centros educativos con los que se había contactado y 29 forman parte del grupo de profesores que comparten ideas en Facebook.

En cuanto al perfil de los docentes (Tabla I) la mayoría son mujeres con una media de edad de 40 años. Respecto al nivel educativo, casi todos son licenciados $(45 \%)$ y con una media de I 3 años de experiencia en la docencia. Destacar que un porcentaje importante $(57 \%)$ imparte su docencia en un centro público en la etapa de Educación Primaria (49\%). El 84 \% nunca ha estado al frente de un aula de Educación Especial.

En relación al rendimiento de los alumnos con discapacidad, el $72 \%$ considera que tienen un rendimiento medio o medio bajo.

\section{Instrumento}

Tras un análisis y revisión de los cuestionarios de actitudes publicados (Larrievé y Cook, I979; García y Alonso, I 985 ; Verdugo et al., I994; Cardona et al., 2000; De Boer et al., 2012) se optó por utilizar para este estudio el cuestionario Actitudes y Prácticas del profesorado relativas a la Inclusión, adaptación realizada por Chiner (20I I), ya que la terminología empleada en él es una de las que más se ajusta a la utilizada en la legislación educativa en España y porque se adapta al objeto de estudio planteado. 


\begin{tabular}{|c|c|c|c|}
\hline & $\begin{array}{c}\text { Colegios ( } n=53) \\
n(\% \text { válido) }\end{array}$ & $\begin{array}{c}\text { Facebook }(n=29) \\
n(\% \text { válido) }\end{array}$ & $\begin{array}{l}\text { Total }(\mathrm{n}=82) \\
\mathrm{n} \text { (\% válido) }\end{array}$ \\
\hline \multicolumn{4}{|l|}{ Género } \\
\hline Varón & $18(34,0 \%)$ & $5(17,2 \%)$ & $23(28,0 \%)$ \\
\hline Mujer & $35(66,0 \%)$ & $24(82,8 \%)$ & $59(72,0 \%)$ \\
\hline \multicolumn{4}{|l|}{ Edad } \\
\hline & $\begin{array}{c}\mathrm{M}=44,32 \\
\mathrm{DT}=10,31 \\
\text { mínimo=25 } \\
\text { máximo=66 }\end{array}$ & $\begin{array}{c}\mathrm{M}=30,39 \\
\mathrm{DT}=6,82 \\
\text { mínimo=22 } \\
\text { máximo }=48\end{array}$ & $\begin{array}{c}\mathrm{M}=39,51 \\
\mathrm{DT}=11,36 \\
\text { mínimo=22 } \\
\text { máximo=66 }\end{array}$ \\
\hline \multicolumn{4}{|l|}{ Nivel de formación } \\
\hline Diplomatura & $22(41,5 \%)$ & $13(44,8 \%)$ & $35(42,7 \%)$ \\
\hline Licenciatura & $26(49,1 \%)$ & $11(37,9 \%)$ & $37(45,1 \%)$ \\
\hline Grado & $3(5,7 \%)$ & $0(0,0 \%)$ & $3(3,7 \%)$ \\
\hline Diplomatura y licenciatura & $2(3,7 \%)$ & $0(0,0 \%)$ & $2(2,4 \%)$ \\
\hline Otros & $0(0,0 \%)$ & $5(17,3 \%)$ & $5(6,1 \%)$ \\
\hline \multicolumn{4}{|l|}{ Años de experiencia } \\
\hline & $\begin{array}{c}\mathrm{M}=18,06 \\
\mathrm{DT}=10,89 \\
\text { mínimo=1 } \\
\text { máximo }=40\end{array}$ & $\begin{array}{c}\mathrm{M}=5,05 \\
\mathrm{DT}=4,66 \\
\text { mínimo=1 } \\
\text { máximo=25 }\end{array}$ & $\begin{array}{c}\mathrm{M}=13,46 \\
\mathrm{DT}=11,02 \\
\text { mínimo=1 } \\
\text { máximo=40 }\end{array}$ \\
\hline \multicolumn{4}{|l|}{ Tipo de centro } \\
\hline Público & $26(49,1 \%)$ & $21(72,4 \%)$ & $47(57,3 \%)$ \\
\hline Concertado & $19(35,8 \%)$ & $6(20,7 \%)$ & $25(30,5 \%)$ \\
\hline Privado & $8(15,1 \%)$ & $2(6,9 \%)$ & $10(12,2 \%)$ \\
\hline \multicolumn{4}{|l|}{ Etapa educativa } \\
\hline Educación Infantil & $7(13,2 \%)$ & $6(20,7 \%)$ & $13(15,9 \%)$ \\
\hline Educación Primaria & $22(41,5 \%)$ & $18(62,1 \%)$ & $40(48,8 \%)$ \\
\hline ESO & $9(17,0 \%)$ & $5(17,2 \%)$ & $14(17,1 \%)$ \\
\hline $\begin{array}{l}\text { Educación Infantil y } \\
\text { Primaria }\end{array}$ & $2(3,8 \%)$ & $0(0,0 \%)$ & $2(2,4 \%)$ \\
\hline Educación Primaria y ESO & $4(7,5 \%)$ & $0(0,0 \%)$ & $4(4,8 \%)$ \\
\hline ESO y Bachillerato & $9(17,0 \%)$ & $0(0,0 \%)$ & $9(11,0 \%)$ \\
\hline \multicolumn{4}{|l|}{ Aula de Educación especial } \\
\hline Sí & $7(13,2 \%)$ & $6(20,7 \%)$ & $13(15,9 \%)$ \\
\hline No & $46(86,8 \%)$ & $23(79,3 \%)$ & $69(84,1 \%)$ \\
\hline \multicolumn{4}{|l|}{ Rendimiento ACNEE } \\
\hline Alto & $2(3,8 \%)$ & $1(3,4 \%)$ & $3(3,7 \%)$ \\
\hline Medio-alto & $3(5,7 \%)$ & $5(17,3 \%)$ & $8(9,8 \%)$ \\
\hline Medio & $19(35,8 \%)$ & $11(37,9 \%)$ & $30(36,6 \%)$ \\
\hline Medio-bajo & $20(37,7 \%)$ & $9(31,0 \%)$ & $29(35,4 \%)$ \\
\hline Bajo & $9(17,0 \%)$ & $3(10,4 \%)$ & $12(14,5 \%)$ \\
\hline
\end{tabular}

Fuente: elaboración propia. 
El instrumento empleado Actitudes y Prácticas del profesorado relativas a la Inclusión es un cuestionario autoadministrado organizado en tres partes: la primera parte relacionada con la Información sociodemográfica, la segunda es el Cuestionario de Percepciones del Profesorado acerca de la Inclusión y la tercera y última parte la Escala de Adaptaciones de la Enseñanza.

La primera parte recoge datos relativos a la edad, género, nivel de formación académica recibida, tipo de centro, materias que imparte (si procede), etapa/as educativa/a, años de experiencia docente, nivel socioeconómico familiar del alumnado, rendimiento académico de sus alumnos con NEE y si han estado al frente de algún aula de Educación Especial.

El cuestionario de la segunda parte evalúa las actitudes del docente hacia la inclusión educativa de niños con NEE (derivadas de discapacidad) en aulas ordinarias. Es un cuestionario compuesto por I 2 ítems organizados en tres categorías que son:

- Bases de la Inclusión (ítems I, 2, 3,4, $5,6,7)$ : mide en qué medida tienen los profesionales actitudes y percepciones positivas hacia los principios filosóficos que sustentan la inclusión. Las puntuaciones oscilan entre 7 y 35 , indicando la puntuación máxima una actitud positiva hacia los principios que defiende la inclusión y la mínima una actitud desfavorable hacia la inclusión de los alumnos con NEE.

- Formación y Recursos (ítems 8, 9, I0): mide las percepciones que tiene el profesorado acerca de su preparación y la disponibilidad de tiempo y recursos materiales para responder a las NEE de sus alumnos. Las puntuaciones varían entre 3 y I 5 , señalando las puntuaciones altas una mayor formación, tiempo y recursos materiales para atender las NEE de los alumnos y las mínimas hacen referencia a que estos profesionales piensan que la formación y los recursos de los que disponen no son suficientes.
- Apoyos Personales (ítems I I, I2): se centra en las percepciones que tienen los profesores sobre el apoyo que reciben por parte de otros profesionales como el profesor de pedagogía terapéutica y el equipo psicopedagógico para atender adecuadamente las necesidades educativas de sus alumnos. Las puntuaciones van desde 2 hasta Io, indicando las puntuaciones más altas una percepción positiva de los docentes respecto a la disponibilidad de apoyos personales y las mínimas una insuficiencia de dichos apoyos.

Es un test de ejecución típica, concretamente una escala tipo Likert de cinco puntos, donde los participantes expresan su opinión sobre cada ítem de acuerdo al siguiente esquema ( $\mathrm{I}=$ NA, nada de acuerdo; $2=\mathrm{PA}$, poco de acuerdo; $3=\mathrm{I}$, indeciso; 4= BA, bastante de acuerdo; 5=MA, muy de acuerdo). La puntuación total oscila entre I 2 (mínima) y 70 (máxima), indicando las puntuaciones altas percepciones y actitudes muy favorables hacia la inclusión y las puntuaciones bajas percepciones y actitudes desfavorables. Se considera un cuestionario fiable $(\alpha=, 69)$.

Por último se utiliza una escala que evalúa las estrategias que utilizan los docentes para favorecer la inclusión educativa de todos sus alumnos en el aula. Esta escala se compone de 2 I ítems organizados en varias categorías de estrategias que son:

- Estrategias de Organización y Manejo Efectivo del Aula (ítems I, 2,3 y 4): examina la frecuencia con que los encuestados utilizan técnicas educativas orientadas a establecer un clima adecuado en el aula y una programación de la enseñanza adaptada al grupo-clase y a las necesidades especiales de algunos alumnos. Las puntuaciones de esta categoría oscilan entre 4 y 20.

- Estrategias de Enseñanza y Evaluación de los Aprendizajes (ítems 5, 6, 7, 8, 9, IO,I I y I2): tratan de favorecer el aprendizaje y la evaluación continua y formativa. La puntuación oscila entre 8 y 40 . 
- Estrategia de Agrupamiento (ítems I3, I4, I 5, I6 y I7): orientadas a flexibilizar la organización del aula mediante la formación de pequeño grupo o pareja para desarrollar determinados aprendizajes. Las puntuaciones oscilan entre 5 y 25 .

- Estrategias de Adaptación de las Actividades (ítems I 8, I9, 20 y 21): conjunto de prácticas educativas diseñadas para acomodar las actividades a las necesidades del grupo o de determinados alumnos, así como el diseño y preparación de materiales específicos y/o alternativos. Las puntuaciones van desde 4 hasta 20.

$\mathrm{Al}$ igual que el cuestionario anterior, es un test de ejecución típica, aunque en este caso, es una escala de tipo graduación de respuesta (ratingscales), con cinco opciones de respuesta que siguen el siguiente esquema $(\mathrm{I}=\mathrm{N}$, nunca; $2=$ $\mathrm{AV}$, a veces; $3=\mathrm{F}$, frecuentemente; $4=\mathrm{CS}$, casi siempre; $5=\mathrm{S}$, siempre). La puntuación total oscila entre 2I (mínima) y Io5 (máxima), refiriéndose las puntuaciones más altas a un mayor uso de estrategias educativas inclusivas y las puntuaciones inferiores a una baja frecuencia en el uso de dichas prácticas. Esta escala es fiable $(\alpha=, 7 \mathrm{I})$.

Respecto al tipo de ítems, se caracterizan por ser afirmaciones sencillas, concisas, breves y sin ambigüedades. Cada ítem aporta información sobre la dimensión o categoría en la que se encuentra y el tiempo que lleva responder al mismo es breve (aproximadamente ro minutos).

\section{Procedimiento}

El procedimiento de recogida de datos se realiza acudiendo a los centros educativos de Salamanca, contactando vía telefónica y correo electrónico en el caso de Zamora y Madrid y vía online a través de un grupo de Facebook.

En primer lugar y referente a los centros educativos, se contacta con el director de cada centro para presentarle el objetivo de la investigación. Se muestra a los centros en el primer contacto una carta de presentación con el propósito del estudio, el instrumento que se va a utilizar y se garantiza la confidencialidad de los datos. Una vez que los centros tomaron la decisión de participar se les pasa el cuestionario junto con la declaración de consentimiento informado.

En el grupo de profesores de Facebook se añade el cuestionario al grupo y se les pide colaboración, explicando con detalle en qué consiste el estudio y los criterios que deben reunir para participar en el mismo.

\section{Análisis estadísticos}

En primer lugar, con el fin de obtener unos resultados adecuados, se llevan a cabo análisis descriptivos de los ítems de cada cuestionario. Se obtiene la distribución de las respuestas de los docentes a cada ítem, así como las medias y desviaciones a cada uno de los ítems y dimensiones.

En segundo lugar, y con el fin de comprobar si los docentes tienen actitudes positivas hacia la inclusión y utilizan estrategias inclusivas, se obtienen las puntuaciones totales en cada dimensión y en las escalas de actitudes y estrategias. A continuación, se comprueban las puntuaciones de nuestra muestra de participantes con los baremos determinados en el cuestionario. Para ello se utilizan pruebas $t$ para una muestra.

En tercer lugar, para demostrar si los docentes que tienen actitudes más positivas usan más estrategias en sus aulas se obtienen correlaciones con el coeficiente de Pearson.

En cuarto lugar, para comprobar la incidencia de las variables sociodemográficas y profesionales sobre las actitudes y estrategias, se realizan los correspondientes contrastes $t$ de diferencia de 
medias para muestras independientes en el caso del género, la edad, nivel de formación y nivel de experiencia, donde únicamente se consideran dos categorías de la variable.

Para las variables edad y años de experiencia se toman dos grupos extremos, por debajo del percentil 25 y por encima del percentil 75 . En relación con la variable edad, el grupo más joven está constituido por aquellos que tienen menos de 29 años, y el grupo de más edad por los docentes con edades superiores a los 48 años. Respecto a los años de experiencia, los de menos experiencia llevan menos de 4 años de docencia, y los de más experiencia más de 22 años.

En el caso de las variables tipo de centro y etapa educativa, se consideran tres categorías y se realizan ANOVAS con las correspondientes pruebas a posteriori. De estos análisis quedan excluidos aquellos docentes que imparten docencia en varias etapas educativas, es decir, únicamente se quedan los maestros que trabajaban individuamente en aulas de Educación Infantil, Primaria y Secundaria.

Para analizar los datos se utiliza el paquete estadístico SPSS, versión 22 y un nivel de significación de $\alpha=0.05$ para considerar un resultado estadísticamente significativo.

\section{Resultados}

Se presentan a continuación los resultados obtenidos. En primer lugar, se analizan las respuestas de los participantes a los diferentes ítems de las escalas de actitudes y estrategias de inclusión.

En el cuestionario de actitudes del profesorado hacia la inclusión, los ítems en los que más alto puntúan los docentes corresponden a la dimensión de bases de inclusión y hacen referencia a que son partidarios de la educación inclusiva, que ésta favorece el desarrollo de actitudes tolerantes y respetuosas entre los alumnos y que para que se dé una respuesta adecuada a la diversidad es importante la presencia en las aulas de otros docentes además del tutor. Se observa que en la dimensión de formación y recursos puntúan más bajo, sobre todo en los ítems que se refieren a la falta de tiempo y de recursos para atender las necesidades de sus alumnos.

En relación a las estrategias inclusivas que utilizan, los ítems con mayor puntuación se relacionan con las dimensiones referentes a las estrategias de organización y manejo efectivo del aula y estrategias de enseñanza y evaluación de los aprendizajes que se refieren a que los docentes establecen normas y rutinas, dedican tiempo a volver a enseñar ciertos conceptos, utilizan estrategias para captar la atención de sus alumnos y motivarles así como llevar un control de su asistencia y progreso.

\section{Actitudes de los docentes hacia la inclusión y estrategias utilizadas}

Una vez realizados los contrastes con los baremos (Tabla 2), se encuentra que los docentes participantes tienen actitudes positivas hacia la inclusión educativa $[\mathrm{t}(8 \mathrm{O})=8,74, \mathrm{p}<, \mathrm{OO} \mathrm{I}]$. En las dimensiones de bases de inclusión $[\mathrm{t}(8 \mathrm{I})=\mathrm{I} 2,82, \mathrm{p}<, \mathrm{OO} \mathrm{I}] \mathrm{y}$ apoyos personales $[\mathrm{t}(80)=2,26, \mathrm{p}=, 027]$ los participantes de nuestro estudio muestran unas actitudes más positivas que el baremo. Sin embargo, en la dimensión de formación y apoyos $[\mathrm{t}(80)=-8,69, \mathrm{p}<, \mathrm{O} \circ \mathrm{I}]$ su actitud es más negativa que el baremo.

Los docentes que participan en el estudio afirman usar significativamente más estrategias inclusivas en sus aulas que el baremo $[\mathrm{t}(79)=\mathrm{I} 7, \mathrm{I} 5, \mathrm{p}<$, ,O I $]$. En los diferentes tipos de estrategias, manifiestan un uso por encima del baremo: estrategias de organización y manejo efectivo del aula $[\mathrm{t}(8 \mathrm{I})=\mathrm{I} 9,95, \mathrm{p}<, \mathrm{OO} \mathrm{I}]$, estrategias de enseñanza y evaluación de los aprendizajes $[\mathrm{t}(79)=20,94, \mathrm{p}<, 00 \mathrm{I}]$, estrategias de agrupamiento $[\mathrm{t}(79)=4,3 \mathrm{I}, \mathrm{p}<$, OOI $] \mathrm{y}$ estrategias de adaptación de actividades $[\mathrm{t}(80)=$ $9,2 \mathrm{I}, \mathrm{p}<, \mathrm{OOI}]$. 


\begin{tabular}{|c|c|c|c|}
\hline \multicolumn{4}{|c|}{$\begin{array}{l}\text { Tabla 2. Descriptivos de las actitudes y las } \\
\text { estrategias del profesorado en la muestra y en } \\
\text { los baremos }\end{array}$} \\
\hline Escalas & Dimensiones & $\begin{array}{c}\text { Participantes } \\
(\mathrm{n}=85)\end{array}$ & Baremo \\
\hline \multirow{4}{*}{\begin{tabular}{|l|} 
Actitudes \\
del \\
profesorado \\
hacia la \\
inclusión
\end{tabular}} & $\begin{array}{l}\text { Bases de } \\
\text { inclusión }\end{array}$ & $\begin{array}{l}27,73 \\
(4,75)\end{array}$ & 21 \\
\hline & $\begin{array}{l}\text { Formación y } \\
\text { recursos }\end{array}$ & $\begin{array}{l}7,91 \\
(2,68)\end{array}$ & 10.5 \\
\hline & \begin{tabular}{|l} 
Apoyos \\
personales
\end{tabular} & $\begin{array}{c}6,58 \\
(2,31)\end{array}$ & 6 \\
\hline & Total & $\begin{array}{l}42,30 \\
(6,49)\end{array}$ & 36 \\
\hline \multirow{5}{*}{$\begin{array}{l}\text { Estrategias } \\
\text { del } \\
\text { profesorado } \\
\text { en inclusión }\end{array}$} & $\begin{array}{l}\text { Estrategias } \\
\text { de } \\
\text { organización } \\
\text { y manejo } \\
\text { efectivo del } \\
\text { aula }\end{array}$ & $\begin{array}{l}16,90 \\
(2,26)\end{array}$ & 12 \\
\hline & $\begin{array}{l}\text { Estrategias } \\
\text { de enseñanza } \\
\text { y evaluación } \\
\text { de los } \\
\text { aprendizajes }\end{array}$ & $\begin{array}{l}34,39 \\
(4,44)\end{array}$ & 24 \\
\hline & $\begin{array}{l}\text { Estrategias } \\
\text { de } \\
\text { agrupamiento }\end{array}$ & $\begin{array}{l}16,74 \\
(3,61)\end{array}$ & 15 \\
\hline & $\begin{array}{l}\text { Estrategias } \\
\text { de } \\
\text { adaptación } \\
\text { de } \\
\text { actividades }\end{array}$ & $\begin{array}{l}15,11 \\
(3,04)\end{array}$ & 12 \\
\hline & Total & $\begin{array}{c}83,20 \\
(10,53)\end{array}$ & 63 \\
\hline
\end{tabular}

Fuente: elaboración propia. resultados y no se podría afirmar rotundamente la relación entre las variables, siendo necesarios estudios posteriores.

Con respecto a esta afirmación, una vez analizadas las correlaciones entre actitudes y estrategias, se comprueba que hay una relación positiva y estadísticamente significativa $[\mathrm{r}(8 \mathrm{O})=, 26, \mathrm{p}=, 02], \mathrm{lo}$ que indicaría que pudiera existir una relación entre tener actitudes positivas hacia la inclusión y utilizar un mayor número de estrategias en las aulas.

Concretamente, la relación es más significativa en el caso de las estrategias de organización y manejo efectivo del aula $[\mathrm{r}(8 \mathrm{I})=, 24, \mathrm{p}=, 029]$ y enseñanza y evaluación $[\mathrm{r}(80)=, 28, \mathrm{p}=$,OI3]. Esto sugiere que podría llegar a existir una relación entre tener actitudes positivas hacia la inclusión y hacer un mayor uso de estrategias que tienen que ver con la organización y manejo del aula y con la enseñanza y evaluación.

Cuando se analizan las diferentes dimensiones de las actitudes, se comprueba que los conocimientos que tienen los docentes sobre la inclusión no se relacionan con un mayor uso de estrategias. Son las dimensiones de formación y recursos $[\mathrm{r}(8 \mathrm{O})=, 35, \mathrm{p}=, \mathrm{OO} \mathrm{I}]$ y apoyos personales $[\mathrm{r}(8 \mathrm{O})=, 3 \mathrm{I}, \mathrm{p}=, 006]$ las que se relacionan más significativamente con el uso de estrategias. Destacar la posible relación significativa que puede llegar haber entre la formación y recursos que tienen los docentes y el uso de estrategias de agrupamiento $[\mathrm{r}(8 \mathrm{O})=, 3 \mathrm{I}, \mathrm{p}=, 005]$; los docentes que consideran que tienen los recursos y el tiempo suficiente serían aquellos que utilizarían más estrategias de agrupamiento, y entre los apoyos personales de los que dispone el docente y las estrategias de organización y manejo del aula $[\mathrm{r}(8 \mathrm{I})=, 34, \mathrm{p}=, 002]$, los profesores que cuentan con la ayuda de otros profesionales especializados serían aquellos que pudieran usar un mayor número de estrategias de organización del aula.

Incidencia de las variables sociodemográficas y profesionales sobre las actitudes

A continuación en la Tabla 3 se muestran los resultados referentes a cómo afectan las variables género, edad, nivel de formación, años 
de experiencia, tipo de centro y etapa educativa en las actitudes de los docentes.

No se encuentran diferencias significativas en las actitudes consideradas globalmente en función del género $[\mathrm{t}(79)=-, 60, \mathrm{p}=, 55 \mathrm{I}]$, ni en las diferentes dimensiones: bases de inclusión $[\mathrm{t}(80)=-, 77, \mathrm{p}=, 447]$, formación y recursos $[\mathrm{t}(79)=, 365, \mathrm{p}=, 7 \mathrm{I} 6]$ y apoyos personales $[\mathrm{t}(79)=$ $-, 355, \mathrm{p}=, 724]$.

Con respecto a la variable edad, tampoco hay diferencias significativas ni en las actitudes consideradas globalmente $[\mathrm{t}(42)=, 97, \mathrm{p}=, 340] \mathrm{ni}$

Tabla 3. Descriptivos en las diferentes dimensiones de la escala de actitudes en función de las variables sociodemográficas y profesionales

\begin{tabular}{|c|c|c|c|c|}
\hline & $\begin{array}{c}\text { Total } \\
\text { M } \\
\text { (DT) }\end{array}$ & $\begin{array}{c}\text { Bases de inclusión } \\
\text { M } \\
\text { (DT) } \\
\end{array}$ & $\begin{array}{c}\text { Formación y recursos } \\
\text { M } \\
\text { (DT) } \\
\end{array}$ & $\begin{array}{c}\text { Apoyos personales } \\
\text { M } \\
\text { (DT) } \\
\end{array}$ \\
\hline \multicolumn{5}{|l|}{ Género } \\
\hline Varón (n=23) & $\begin{array}{l}41,61 \\
(5,61) \\
\end{array}$ & $\begin{array}{l}27,09 \\
(5,61) \\
\end{array}$ & $\begin{array}{c}8,09 \\
(2,27) \\
\end{array}$ & $\begin{array}{c}6,43 \\
(2,25) \\
\end{array}$ \\
\hline Mujer $(n=58)$ & $\begin{array}{l}42,57 \\
(6,83)\end{array}$ & $\begin{array}{l}27,98 \\
(4,81)\end{array}$ & $\begin{array}{c}7,84 \\
(2,84)\end{array}$ & $\begin{array}{c}6,64 \\
(2,35)\end{array}$ \\
\hline \multicolumn{5}{|l|}{ Edad } \\
\hline >29 años (n=23) & $\begin{array}{l}43,70 \\
(6,43)\end{array}$ & $\begin{array}{l}29,35 \\
(4,13)\end{array}$ & $\begin{array}{c}7,7 \\
(2,92)\end{array}$ & $\begin{array}{c}6,57 \\
(2,46)\end{array}$ \\
\hline$>48$ años $(n=21)$ & $\begin{array}{l}41,57 \\
(8,15)\end{array}$ & $\begin{array}{l}26,76 \\
(6,04) \\
\end{array}$ & $\begin{array}{c}8,24 \\
(2,58) \\
\end{array}$ & $\begin{array}{c}6,57 \\
(2,58) \\
\end{array}$ \\
\hline \multicolumn{5}{|l|}{ Nivel de formación } \\
\hline Diplomatura $(n=34)$ & $\begin{array}{l}41,03 \\
(6,74) \\
\end{array}$ & $\begin{array}{l}27,03 \\
(4,97) \\
\end{array}$ & $\begin{array}{c}7,09 \\
(2,95)\end{array}$ & $\begin{array}{c}6,77 \\
(2,56) \\
\end{array}$ \\
\hline Licenciatura $(n=37)$ & $\begin{array}{l}42,86 \\
(6,73) \\
\end{array}$ & $\begin{array}{l}28,14 \\
(4,69) \\
\end{array}$ & $\begin{array}{c}8,43 \\
(2,40) \\
\end{array}$ & $\begin{array}{c}6,30 \\
(2,30) \\
\end{array}$ \\
\hline \multicolumn{5}{|l|}{ Años de experiencia } \\
\hline < 4años $(\mathrm{n}=22)$ & $\begin{array}{l}42,36 \\
(6,93) \\
\end{array}$ & $\begin{array}{l}28,68 \\
(3,96) \\
\end{array}$ & $\begin{array}{c}7,27 \\
(3,13) \\
\end{array}$ & $\begin{array}{c}6,41 \\
(2,56) \\
\end{array}$ \\
\hline >22 años (n=21) & $\begin{array}{l}42,19 \\
(8,17)\end{array}$ & $\begin{array}{l}27,14 \\
(6,14)\end{array}$ & $\begin{array}{c}8,67 \\
(2,33)\end{array}$ & $\begin{array}{l}6,38 \\
(2,69 \\
\end{array}$ \\
\hline \multicolumn{5}{|l|}{ Tipo de centro } \\
\hline Público (n=46) & $\begin{array}{l}42,44 \\
(7,00)\end{array}$ & $\begin{array}{l}28,66 \\
(4,33) \\
\end{array}$ & $\begin{array}{c}7,30 \\
(2,86) \\
\end{array}$ & $\begin{array}{c}6,33 \\
(2,43) \\
\end{array}$ \\
\hline Concertado(n=25) & $\begin{array}{l}42,68 \\
(6,24) \\
\end{array}$ & $\begin{array}{l}25,92 \\
(5,43) \\
\end{array}$ & $\begin{array}{r}9,08 \\
(2,22) \\
\end{array}$ & $\begin{array}{c}7,68 \\
(1,80) \\
\end{array}$ \\
\hline Privado $(n=10)$ & $\begin{array}{c}40,7 \\
(4,72) \\
\end{array}$ & $\begin{array}{l}27,90 \\
(3,93) \\
\end{array}$ & $\begin{array}{c}7,80 \\
(2,04) \\
\end{array}$ & $\begin{array}{c}5,00 \\
(1,70) \\
\end{array}$ \\
\hline \multicolumn{5}{|l|}{ Etapa educativa } \\
\hline E.I $(n=13)$ & $\begin{array}{l}41,08 \\
(4,80) \\
\end{array}$ & $\begin{array}{l}28,23 \\
(4,73) \\
\end{array}$ & $\begin{array}{c}7,00 \\
(2,65) \\
\end{array}$ & $\begin{array}{c}5,85 \\
(2,27) \\
\end{array}$ \\
\hline E.P $(n=39)$ & $\begin{array}{l}44,00 \\
(7,11) \\
\end{array}$ & $\begin{array}{l}28,20 \\
(4,30) \\
\end{array}$ & $\begin{array}{c}8,39 \\
(2,93) \\
\end{array}$ & $\begin{array}{c}7,26 \\
(2,11) \\
\end{array}$ \\
\hline $\mathrm{ESO}(n=14)$ & $\begin{array}{l}38,50 \\
(5,80)\end{array}$ & $\begin{array}{l}25,50 \\
(6,10)\end{array}$ & $\begin{array}{c}6,86 \\
(2,48)\end{array}$ & $\begin{array}{c}6,14 \\
(2,48)\end{array}$ \\
\hline
\end{tabular}

Fuente: elaboración propia. 
en cada una de las categorías: bases de inclusión $\left[\mathrm{t}(42)=\mathrm{I}, 67, \mathrm{p}=, \mathrm{IO}_{2}\right]$, formación y recursos $[\mathrm{t}(42)=-, 55, \mathrm{p}=, 587]$ y apoyos personales $[\mathrm{t}(42)=$ $-, 008, p=, 994]$. Aunque las diferencias no son significativas, los docentes más jóvenes tienen actitudes más positivas hacia la inclusión que los profesores de más edad. Además, son estos mismos los que afirman conocer con más detalle la filosofía de la inclusión.

En relación a la variable nivel de formación, las diferencias en las actitudes no son significativas ni globalmente $[\mathrm{t}(69)=-\mathrm{I}, \mathrm{I} 5, \mathrm{p}=, 255]$, ni en las dimensiones de bases de inclusión [ $\mathrm{t}(70)=-$ $, 97, p=, 335]$ y apoyos personales $[t(69)=, 8 \mathrm{I}$, $\mathrm{p}=, 420]$. Aun así, los docentes que poseen una licenciatura tienen actitudes más favorables hacia la educación inclusiva y conocen mejor las bases de la inclusión que los docentes que tienen una formación de tres años (una diplomatura). Encontramos únicamente diferencias significativas en la dimensión de formación y recursos $[\mathrm{t}(69)=-2, \mathrm{I} 2, \mathrm{p}=, 038]$, en la que los docentes con una licenciatura tienen actitudes más positivas hacia la inclusión que aquellos que tienen una diplomatura.

En lo que se refiere a la variable de años de experiencia, las diferencias no son significativas ni de manera global $[\mathrm{t}(4 \mathrm{I})=, 08, \mathrm{p}=, 94 \mathrm{I}] \mathrm{ni}$ en ninguna de las tres dimensiones: bases de inclusión [ $\mathrm{t}(4 \mathrm{I})=, 98, \mathrm{p}=, 332]$, formación $y$ recursos $[\mathrm{t}(4 \mathrm{I})=-\mathrm{I}, 65, \mathrm{p}=$, IO 7$]$ y apoyos personales $[\mathrm{t}(4 \mathrm{I})=, 04, \mathrm{p}=, 972]$. A pesar de no ser significativas, los docentes con menos años de experiencia tienen actitudes más favorables en la dimensión de bases de inclusión que aquellos docentes con más experiencia. Aunque en el caso de la dimensión de formación y recursos ocurre lo contrario, los docentes con más experiencia afirman que tienen más formación y recursos que los que tienen menos experiencia.

En lo que respecta al tipo de centro, las diferencias no son significativas en las actitudes ni globalmente $[\mathrm{F}(2,78)=, 35, \mathrm{p}=, 705]$, ni en la dimensión de bases de inclusión $[\mathrm{F}(2,79)=2,84$, $\mathrm{p}=, 064]$. Aun así, los docentes de los colegios concertados y públicos tienen actitudes más positivas que los de los centros privados. Sin embargo, los resultados recogen que son los profesores de los colegios concertados los que tienen menos conocimientos sobre las bases de la inclusión que los profesores que ejercen su labor en centros públicos y privados. Las diferencias sí son significativas en la dimensión de formación $y$ recursos $\left[\mathrm{F}(2,78)=3,82, \mathrm{p}=, 026, \eta^{2}=, 089\right]$ y apoyos personales $[\mathrm{F}(2,78)=6, \mathrm{I} 4, \mathrm{p}=, 003$, $\left.\eta^{2}=, \mathrm{I} 36\right]$. Al realizar los contrastes a posteriori, sólo se hallan diferencias estadísticamente significativas entre los colegios públicos y concertados $(\mathrm{p}=, 026)$ en lo que respecta a la formación y recursos, lo cual quiere decir que los profesores de los centros concertados afirman tener más formación y recursos para atender las necesidades de sus alumnos que los profesores de los centros públicos. También se hayan diferencias significativas entre los colegios concertados y privados en la dimensión de apoyos personales $(\mathrm{p}=, 006)$, es decir, el profesorado de los centros concertados consideran que cuentan con un mayor número de apoyos personales que los docentes de los centros privados.

Por último, en la variable de etapa educativa sólo se encuentran diferencias significativas en las actitudes globalmente $[\mathrm{F}(2,63)=4,00$, $\left.\mathrm{p}=, 023, \eta^{2}=, \mathrm{II}\right]$. Con las pruebas a posteriori se comprueba que las diferencias estaban entre la etapa de la ESO y Primaria $(\mathrm{p}=, 03)$, es decir, los docentes de Primaria tienen actitudes más positivas hacia la educación inclusiva que los profesores de la ESO. Las diferencias no son significativas en bases de inclusión $[\mathrm{F}(2,64)=\mathrm{I}, 77, \mathrm{p}=, \mathrm{I} 79]$, formación y recursos $[\mathrm{F}(2,63)=2,20, \mathrm{p}=, \mathrm{I} 2 \mathrm{O}]$ y recursos personales $[\mathrm{F}(2,63)=2,6 \mathrm{I}, \mathrm{p}=, 08 \mathrm{I}]$. Aunque las diferencias en estas dimensiones no son significativas, los docentes de las etapas educativas de Infantil y Primaria afirman tener más nociones de la inclusión, así como mayor formación y recursos que los docentes de la ESO. Sin embargo, en apoyos personales, los docentes de Infantil son los que menos apoyos tienen en relación a las otras dos etapas educativas.

Por lo tanto, los resultados obtenidos demuestran que las variables profesionales referentes al nivel de formación, tipo de 
centro y etapa educativa son las que tienen una mayor incidencia en las actitudes que las variables sociodemográficas. Concretamente, los maestros que imparten docencia en la etapa de Educación Primaria son los que tienen actitudes más positivas hacia la inclusión. Además, los profesores de los centros concertados son los que afirman tener un mayor número de apoyos personales que infiere en una actitud más positiva hacia la inclusión. Por último, los docentes de centros concertados y con una licenciatura afirman tener una mayor formación y disponen de más recursos.

Incidencia de las variables sociodemográficas y profesionales en las estrategias

En la Tabla 4 se detalla la incidencia de las variables de edad, género, nivel de formación, años de experiencia, tipo de centro y etapa educativa sobre las diferentes estrategias que usan los docentes en sus aulas.

Se encuentran diferencias significativas en función del género en la utilización de estrategias $[\mathrm{t}(78)=-$ I,69, p=,094]. Concretamente, en relación a las diferentes estrategias, las diferencias no son significativas en lo referente a enseñanza y evaluación de los aprendizajes $[\mathrm{t}(78)=-\mathrm{I}, 5 \mathrm{I}$, $\mathrm{p}=, \mathrm{I} 35]$ y estrategias de agrupamiento $[\mathrm{t}(78)=$ $-, 02, p=, 988]$. Por el contrario, sí son diferencias significativas en las estrategias de organización y manejo efectivo del aula $[\mathrm{t}(8 \mathrm{O})=-2,24, \mathrm{p}=, 028]$ $\mathrm{y}$ estrategias de adaptación de actividades $[\mathrm{t}(79)=$ $-2,20, p=, 03 \mathrm{I}]$, aunque como se puede observar son las mujeres las que más utilizan todos los tipos de estrategias principalmente las de adaptación de actividades.

Respecto a la edad, las diferencias sólo son significativas en las estrategias de organización y manejo efectivo del aula $[\mathrm{t}(42)=2,30, \mathrm{p}=, 026]$, las cuales son utilizadas más por los docentes más jóvenes. Por lo tanto, de manera global $\left[\mathrm{t}(42)=\mathrm{I}, 02, \mathrm{p}=, 3 \mathrm{I}_{5}\right]$ y en las estrategias de enseñanza y evaluación de los aprendizajes $[t(42)=, 53, p=, 597]$, estrategias de agrupamiento $[\mathrm{t}(42)=, 8 \mathrm{I}, \mathrm{p}=, 425]$ y estrategias de adaptación de las actividades $[\mathrm{t}(42)=, 07, \mathrm{p}=, 949]$ las diferencias no son significativas, aunque todas son utilizadas más por los docentes jóvenes.

Referente al nivel de formación, las diferencias son significativas a nivel global $[\mathrm{t}(68)=, 2 \mathrm{I}$, $\mathrm{p}=, 045]$. Los docentes con formación de diplomatura utilizan globalmente más estrategias que los de licenciatura. Sin embargo, cuando se analizan las distintas estrategias las diferencias no son significativas en las estrategias relativas a organización y manejo efectivo $[\mathrm{t}(7 \mathrm{O})=\mathrm{I}, 37, \mathrm{p}=, \mathrm{I} 74]$, de agrupamiento $[\mathrm{t}(68)=, 73, \mathrm{p}=, 466]$ y adaptación de actividades $[\mathrm{t}(69)=\mathrm{I}, 68, \mathrm{p}=, 098]$. En las estrategias de enseñanza y evaluación de los aprendizajes sí son significativas $[\mathrm{t}(68)=2,43, \mathrm{p}=$, OI 8$]$, siendo los docentes que tienen una diplomatura los que usan más este tipo de estrategias que aquellos que tienen una licenciatura.

Respecto a los años de experiencia, cuando se analizan a nivel global las estrategias, las diferencias no son significativas $[\mathrm{t}(4 \mathrm{I})=\mathrm{I}, 2$, $\mathrm{p}=, 223$ ], sin embargo cuando se analizan individualmente, aquellos con menos años de experiencia usan más estrategias de organización y manejo efectivo del aula que los que llevan trabajando más de 22 años, siendo éstas diferencias significativas $[\mathrm{t}(4 \mathrm{I})=2,24$, $\mathrm{p}=, 03 \mathrm{I}]$. En el resto de estrategias, también son los que menos experiencia tienen los que más las utilizan, pero las diferencias no son significativas: estrategias de enseñanza y evaluación de los aprendizajes [ $\mathrm{t}(4 \mathrm{I})=, 64$, $\mathrm{p}=, 528]$, estrategias de agrupamiento $[\mathrm{t}(4 \mathrm{I})=\mathrm{I}, \mathrm{\circ}, \mathrm{p}=, 323]$ y estrategias de adaptación de actividades $[t(4 \mathrm{I})=, 4 \mathrm{I}, \mathrm{p}=, 682]$.

Cuando se analiza el tipo de centro, únicamente en las estrategias de agrupamiento $[\mathrm{F}(2,77)=\mathrm{I}, 4 \mathrm{O}, \mathrm{p}=, 253]$ las diferencias no son significativas. Sin embargo sí hay diferencias significativas en las estrategias de manera global $\left[\mathrm{F}(2,77)=6,65, \mathrm{p}=, 002, \eta^{2}=, \mathrm{I} 47\right]$. Tras realizar las pruebas a posteriori, se comprueba que estas diferencias están entre los centros privados y públicos $(\mathrm{p}=, 005)$ y entre los centros privados $\mathrm{y}$ concertados $(\mathrm{p}=, 003)$, siendo los docentes de los centros públicos y concertados los que usan más estrategias inclusivas que los de los privados. 
Tabla 4. Descriptivos en las diferentes dimensiones de la escala de estrategias en función de las variables sociodemográficas y profesionales

\begin{tabular}{|c|c|c|c|c|c|}
\hline & $\begin{array}{c}\text { Total } \\
\text { M } \\
\text { (DT) }\end{array}$ & $\begin{array}{c}\text { Estrategias } \\
\text { organización y } \\
\text { manejo del aula } \\
\text { M } \\
\text { (DT) }\end{array}$ & $\begin{array}{c}\text { Estrategias enseñanza } \\
\text { y evaluación de } \\
\text { aprendizajes } \\
\text { M } \\
\text { (DT) }\end{array}$ & $\begin{array}{c}\text { Estrategias } \\
\text { agrupamiento } \\
M \\
\text { (DT) }\end{array}$ & $\begin{array}{c}\text { Estrategias } \\
\text { adaptación } \\
\text { actividades } \\
\text { M } \\
\text { (DT) }\end{array}$ \\
\hline \multicolumn{6}{|l|}{ Género } \\
\hline Varón (n=22) & $\begin{array}{c}80,00 \\
(11,35)\end{array}$ & $\begin{array}{l}16,04 \\
(2,10)\end{array}$ & $\begin{array}{l}33,18 \\
(4,81)\end{array}$ & $\begin{array}{l}16,73 \\
(3,76)\end{array}$ & $\begin{array}{l}13,96 \\
(3,21)\end{array}$ \\
\hline Mujer $(n=58)$ & $\begin{array}{c}84,41 \\
(10,04)\end{array}$ & $\begin{array}{l}17,24 \\
(2,20)\end{array}$ & $\begin{array}{l}34,84 \\
(4,24)\end{array}$ & $\begin{array}{l}16,74 \\
(3,59)\end{array}$ & $\begin{array}{l}15,57 \\
(2,87)\end{array}$ \\
\hline \multicolumn{6}{|l|}{ Edad } \\
\hline >29 años (n=23) & $\begin{array}{l}84,52 \\
(8,60)\end{array}$ & $\begin{array}{l}17,43 \\
(1,90)\end{array}$ & $\begin{array}{l}34,91 \\
(4,07)\end{array}$ & $\begin{array}{l}16,78 \\
(2,68)\end{array}$ & $\begin{array}{l}15,39 \\
(2,59)\end{array}$ \\
\hline >48 años ( $\mathrm{n}=21)$ & $\begin{array}{c}81,38 \\
(11,77)\end{array}$ & $\begin{array}{l}15,91 \\
(2,49)\end{array}$ & $\begin{array}{l}34,19 \\
(4,92)\end{array}$ & $\begin{array}{l}15,95 \\
(4,08)\end{array}$ & $\begin{array}{l}15,33 \\
(3,35)\end{array}$ \\
\hline \multicolumn{6}{|l|}{ Nivel de formación } \\
\hline $\begin{array}{l}\text { Diplomatura } \\
(\mathrm{n}=34)\end{array}$ & $\begin{array}{l}85,00 \\
(9,63)\end{array}$ & $\begin{array}{l}17,20 \\
(2,19)\end{array}$ & $\begin{array}{l}35,50 \\
(4,29)\end{array}$ & $\begin{array}{l}16,71 \\
(3,26)\end{array}$ & $\begin{array}{l}15,56 \\
(2,80)\end{array}$ \\
\hline $\begin{array}{l}\text { Licenciatura } \\
(\mathrm{n}=36)\end{array}$ & $\begin{array}{c}79,94 \\
(10,94)\end{array}$ & $\begin{array}{l}16,46 \\
(2,38)\end{array}$ & $\begin{array}{l}32,97 \\
(4,40)\end{array}$ & $\begin{array}{l}16,08 \\
(3,81)\end{array}$ & $\begin{array}{l}14,35 \\
(3,23)\end{array}$ \\
\hline \multicolumn{6}{|c|}{ Años de experiencia } \\
\hline < 4años $(\mathrm{n}=22)$ & $\begin{array}{c}83,59 \\
(10,24)\end{array}$ & $\begin{array}{l}17,50 \\
(2,22)\end{array}$ & $\begin{array}{l}34,59 \\
(4,43)\end{array}$ & $\begin{array}{l}16,50 \\
(3,16)\end{array}$ & $\begin{array}{l}15,00 \\
(2,96)\end{array}$ \\
\hline$>22$ años $(n=21)$ & $\begin{array}{l}79,71 \\
(10,28\end{array}$ & $\begin{array}{l}15,90 \\
(2,45)\end{array}$ & $\begin{array}{l}33,71 \\
(4,62)\end{array}$ & $\begin{array}{l}15,48 \\
(3,54)\end{array}$ & $\begin{array}{l}14,62 \\
(3,09)\end{array}$ \\
\hline \multicolumn{6}{|l|}{ Tipo de centro } \\
\hline Público ( $\mathrm{n}=45)$ & $\begin{array}{c}84,20 \\
(10,19)\end{array}$ & $\begin{array}{l}17,02 \\
(2,13)\end{array}$ & $\begin{array}{l}35,07 \\
(4,37)\end{array}$ & $\begin{array}{l}16,69 \\
(3,38)\end{array}$ & $\begin{array}{l}15,30 \\
(3,10)\end{array}$ \\
\hline Concertado $(n=25)$ & $\begin{array}{l}85,60 \\
(7,64)\end{array}$ & $\begin{array}{l}17,40 \\
(1,96)\end{array}$ & $\begin{array}{l}34,92 \\
(3,38)\end{array}$ & $\begin{array}{l}17,44 \\
(3,90)\end{array}$ & $\begin{array}{l}15,84 \\
(2,30)\end{array}$ \\
\hline Privado $(n=10)$ & $\begin{array}{c}72,70 \\
(12,94)\end{array}$ & $\begin{array}{l}15,10 \\
(2,60)\end{array}$ & $\begin{array}{l}30,00 \\
(4,97)\end{array}$ & $\begin{array}{l}15,20 \\
(3,74)\end{array}$ & $\begin{array}{l}12,40 \\
(3,20)\end{array}$ \\
\hline \multicolumn{6}{|l|}{ Etapa educativa } \\
\hline E.I $(n=13)$ & $\begin{array}{c}84,08 \\
(11,38)\end{array}$ & $\begin{array}{l}17,62 \\
(2,06)\end{array}$ & $\begin{array}{l}34,39 \\
(5,33)\end{array}$ & $\begin{array}{l}16,54 \\
(3,78)\end{array}$ & $\begin{array}{l}15,54 \\
(2,57)\end{array}$ \\
\hline E.P $(n=38)$ & $\begin{array}{l}86,37 \\
(9,38) \\
\end{array}$ & $\begin{array}{l}17,48 \\
(1,99)\end{array}$ & $\begin{array}{l}35,34 \\
(4,19)\end{array}$ & $\begin{array}{l}17,76 \\
(3,02)\end{array}$ & $\begin{array}{l}15,62 \\
(37), 0\end{array}$ \\
\hline ESO $(n=14)$ & $\begin{array}{l}82,71 \\
(6,84)\end{array}$ & $\begin{array}{l}16,71 \\
(1,49)\end{array}$ & $\begin{array}{l}34,86 \\
(2,03)\end{array}$ & $\begin{array}{l}16,29 \\
(4,14)\end{array}$ & $\begin{array}{l}14,86 \\
(2,11)\end{array}$ \\
\hline
\end{tabular}

Fuente: elaboración propia. 
En las estrategias de organización y manejo efectivo del aula $\left[\mathrm{F}(2,79)=4,29, \mathrm{p}=, 0 \mathrm{I} 7, \eta^{2}=, 098\right]$ las diferencias son significativas entre los colegios privados y públicos $(\mathrm{p}=, 04 \mathrm{I})$ y privados y concertados $(\mathrm{p}=, 020)$, donde los centros privados usan menos este tipo de estrategias que los otros dos tipos de centros. En las estrategias de enseñanza y evaluación de los aprendizajes las diferencias son significativas $[\mathrm{F}(2,77)=6,36$, $\left.\mathrm{p}=, 003, \eta^{2}=, \mathrm{I} 42\right]$, se comprueba que las diferencias siguen estando entre los mismos centros como en los casos anteriores, centros privados y públicos $(\mathrm{p}=, 004)$ y privados y concertados $(\mathrm{p}=, 009)$, de los cuales los públicos y concertados usan más estrategias de este tipo que los privados. Por último, en las estrategias de adaptación de actividades las diferencias también son significativas $[\mathrm{F}(2,78)=5,30$, $\left.\mathrm{p}=, 007, \eta^{2}=, \mathrm{I} 2 \mathrm{O}\right]$. Las pruebas a posteriori demuestran que estas diferencias están al igual que en las estrategias anteriores entre los centros privados y públicos ( $\mathrm{p}=$,oI9) y los privados y concertados $(\mathrm{p}=, 009)$, siendo de nuevo los colegios privados los que usan menos estrategias de este tipo que los concertados y públicos.

Por último, en relación a la etapa educativa en la que imparten docencia los profesores participantes en este trabajo se encuentra que no hay diferencias en las estrategias consideradas globalmente $[\mathrm{F}(2,62)=, 88, \mathrm{p}=, 4 \mathrm{I} 8]$, ni en las diferentes estrategias: organización y manejo efectivo del aula $[\mathrm{F}(2,64)=, 98, \mathrm{p}=, 379]$, enseñanza y evaluación de los aprendizajes $[\mathrm{F}(2,62)=, 28, \mathrm{p}=, 755]$, agrupamiento $[\mathrm{F}(2,62)=\mathrm{I}, 25, \mathrm{p}=, 294]$ y adaptación de actividades $[\mathrm{F}(2,63)=, 39, \mathrm{p}=, 68 \mathrm{I}]$. Aunque las diferencias no son significativas sí se puede señalar de manera general que los docentes de las etapas educativas de Infantil y Primaria usan más estrategias que los docentes de nuestro estudio que imparten docencia en la ESO.

Por lo tanto, los resultados en relación a la incidencia de las variables sociodemográficas y profesionales en las estrategias muestran que los docentes de centros públicos o concertados son los que utilizan más estrategias inclusivas. Concretamente, las profesoras de menos de 29 años con una experiencia menor de cuatro años y que imparten docencia en centros públicos o privados son las que más utilizan estrategias de organización y manejo efectivo del aula. Respecto a las estrategias de enseñanza y evaluación de los aprendizajes, son más utilizadas por docentes que desarrollan su labor en centros públicos o concertados y que tienen una diplomatura. A su vez, las estrategias de adaptación de actividades son utilizadas sobre todo por las profesoras de centros públicos o concertados. Finalmente, en las estrategias de agrupamiento las diferencias no son significativas para ninguna de las variables analizadas.

\section{Discusión y conclusiones}

Los cambios que se están produciendo en la atención a la diversidad conllevan conocer e investigar si las propuestas metodológicas y organizativas junto a las actitudes del profesorado están respondiendo a la realidad de las aulas. El trabajo que se presenta aporta interesantes resultados en este ámbito de investigación en el que, como se ha podido comprobar en el análisis efectuado, hay escasez de estudios. Se obtienen aportaciones originales al tema estudiado a través de un análisis empírico con datos reales. Se presentan a continuación los aspectos más importantes derivados de los resultados de este estudio.

Los docentes participantes en el estudio muestran una actitud positiva hacia la inclusión educativa de alumnos con NEE derivadas de discapacidad en las aulas. Estos resultados coinciden con los obtenidos en otros trabajos (Alemany y Villuendas, 2004; Álvarez et al., 2008; Avramidis y Norwich, 2002; Bradfield et al., I973; Cook et al., 2007; Higgs, I975; Nicasio y Alonso, I 985 ; Van Reusen et al., 2000; Vicuña, 20I3; Scruggs y Mastropieri, I996) que afirman que los docentes están de acuerdo con los principios de la inclusión y los beneficios que produce para todos los alumnos. 
En relación a las estrategias, que es el otro punto fuerte del estudio, se han obtenido resultados similares con los estudios realizados por Chiner (20II) y Vicuña (20I3), los cuales afirman que los docentes hacen a diario un uso moderado de estrategias y prácticas inclusivas en sus aulas. Principalmente utilizan estrategias de organización y manejo efectivo del aula, seguido de estrategias de enseñanza y evaluación y adaptación de actividades y en menor medida estrategias de agrupamiento. Esto puede ser debido a que las estrategias más utilizadas tienen que ver con aquellas que requieren de menos tiempo de preparación y menos recursos tanto materiales como personales.

Más concretamente, en cuanto a los resultados obtenidos en relación a si hay variables que influyen en las actitudes que tengan los docentes, se ha podido comprobar que la edad, el género y el nivel de formación no influyen en las actitudes de los docentes. En trabajos como el de Avramidis et al. (2000), Harasymiw y Horne (I975), Nicasio y Alonso (I985) y Vicuña (20I3) no se encontraron diferencias significativas en estas variables con tener una actitud favorable hacia la inclusión educativa. No obstante, estos estudios concuerdan con los resultados que se han obtenido en relación a que si es cierto que aunque estas diferencias no son significativas, se ha podido apreciar como son las mujeres, los docentes de menor edad y con un nivel de formación de licenciatura los que afirman tener actitudes más positivas hacia la inclusión. Sin embargo, también hay estudios que contradicen esta idea, como se puede apreciar en los resultados obtenidos de investigaciones realizadas anteriormente (Abós y Polaino, I986; Alemany y Villuendas, 2004; Flores, 2007; Suriá, 2012) en las que se afirma que el género sí infiere en las actitudes, de tal manera que son los varones los que tienen actitudes más positivas hacia la inclusión que las mujeres. No obstante, estas investigaciones también demostraron que la edad no influye significativamente y que son los de menor edad los que tienen actitudes más positivas. Además, los docentes con menos experiencia son los que tienen mejores actitudes hacia la educación inclusiva (Avramidis et al., 2000; Avramidis y
Norwich, 2002; Dendra et al., I99I; Granada et al., 2013; Harasymiw y Horne, I975; McCauley et al., I978; Padeliadu y Lampropoulou, I997; Van Reusen et al., 2000). Sin embargo, Vicuña (20I3) en su investigación señala lo contrario, que son los docentes con más años de experiencia los que tienen una actitud más favorable.

Nicasio y Alonso (I985) señalan en relación a las variables relacionadas con el tipo de centro y etapa educativa que no hay diferencias significativas. En general, los docentes de los centros públicos o concertados tienen actitudes más favorables que los de los centros privados. La variable etapa educativa influye en las actitudes de los docentes, aunque los estudios que hay al respecto son contradictorios. Las investigaciones de Chiner (20II), Nicasio y Alonso (I985) y Suriá (20I2) afirman que los docentes de las etapas educativas de Infantil y Primaria tienen actitudes más positivas que los de Educación Secundaria. A su vez, Cardona (2004) y Savage y Wienke (I989) indican que a medida que aumenta la etapa educativa, los docentes muestran actitudes más negativas hacia la inclusión. Esto puede ser debido a que los docentes de etapas educativas superiores se centran más en los objetivos y contenidos mínimos curriculares que en contenidos y competencias transversales poniendo en el centro del proceso de enseñanza los mínimos establecidos y no al alumnado. Sin embargo, Avramidis y Norwich (2002) demostraron lo contrario, es decir, que son los docentes de la ESO los que tienen actitudes más favorables.

Chiner (20I I) afirma que el género y el nivel de formación no se relacionan con un mayor uso de estrategias inclusivas. Sin embargo, aunque las diferencias no son significativas, son las mujeres y los docentes con estudios de licenciatura los que utilizan más estrategias. En relación al tipo de centro, los públicos y concertados implementan un mayor número de estrategias que los privados.

En lo referente a la relación que se establece entre etapa educativa y uso de estrategias inclusivas, cabe destacar que en esta 
investigación no se encontró una relación entre ambas variables como en estudios anteriores que sí demostraron que los docentes de las etapas educativas de Infantil y Primaria son los que usan más estrategias que los de la ESO (Cardona et al., 2003; McIntosh et al., I994).

Por último, los resultados concuerdan con otras investigaciones que afirman que tener una actitud más positiva hacia la inclusión se relaciona con un mayor uso de estrategias inclusivas en las aulas (Ballone y Czerniak, 200I; Leyser y Tappendorf, 200I).

Teniendo en cuenta los resultados de este estudio se determina (aunque no se pueden generalizar los resultados al obtenerse correlaciones bajas) que los docentes tienen actitudes positivas hacia la inclusión educativa aunque precisan de mayor formación y recursos personales. Los docentes hacen un uso frecuente de estrategias inclusivas en sus aulas, sobre todo aquellas que tienen que ver con la organización y manejo del aula y enseñanza y evaluación de los aprendizajes. Tanto las actitudes como las estrategias varían sobre todo en relación a variables relacionadas con la práctica profesional y no tanto con variables sociodemográficas. Finalmente, hay una relación directa entre tener actitudes positivas hacia la inclusión y utilizar un mayor número de estrategias en las aulas.

En conclusión, los resultados de este estudio son de interés y de relevancia para profundizar y conocer las implicaciones y las demandas que precisan los docentes para adquirir actitudes positivas y usar más estrategias inclusivas, ya que el papel de los profesores es parte importante de la calidad educativa y del desarrollo integral del alumnado. Básicamente estas demandas se refieren a la formación y recursos personales y materiales. Por ello, hay que hacer hincapié en estos aspectos para lograr el éxito de todos los alumnos en su proceso de enseñanza-aprendizaje y que todos tengan una educación de calidad formándose como ciudadanos del siglo XXI.

El estudio realizado presenta algunas limitaciones. A día de hoy, los términos inclusión educativa, educación inclusiva y necesidades educativas especiales aún producen confusiones conceptuales lo cual implica y no se puede descartar que los docentes encuestados tengan un concepto de estos términos diferente al utilizado en este trabajo debido en parte a su formación. En los casos de profesores con más años de experiencia se les formó en modelos y estrategias de intervención centradas en la filosofía integradora alejados, en aquel momento, de las estrategias y metodologías centradas en el alumno, en la participación, en las capacidades de cada uno y en transformar los centros en centros inclusivos, principios de la educación inclusiva.

Investigaciones futuras pueden encaminarse a conocer qué programas de formación (inicial y continua) y apoyos hay en los centros, examinar si son eficaces para el cambio de actitudes de los profesionales del ámbito educativo y adquieran actitudes positivas hacia la diversidad y la inclusión de todos en todos los contextos y observar e investigar si mayor y mejor formación implica un mayor y mejor uso de metodología y prácticas inclusivas. Comprobar si la actitud de los docentes hacia la inclusión repercute o no en el proceso de enseñanza-aprendizaje de sus alumnos y al mismo tiempo profundizar en las estrategias que los centros están implementando para atender a la diversidad y si influyen en las actitudes de los profesionales.

Otra limitación es el tamaño de la muestra, el objetivo de próximos trabajos es ampliarla para eliminar esta dificultad y así poder generalizar los resultados. Se ampliará con la inclusión de otros profesionales del ámbito educativo (equipo psicopedagógico, docentes de apoyo, de educación especial, de audición y lenguaje, educadores sociales...) y se incluirá a los alumnos, con y sin discapacidad, con el fin de conocer su percepción respecto a las estrategias inclusivas desarrolladas en sus centros y relacionar las actitudes con dichas percepciones, aspectos que no han sido objeto de estudio en esta investigación.

En posteriores trabajos se ampliará y se utilizará una metodología tanto cuantitativa 
como cualitativa, se realizarán otros análisis que complementen los realizados incluyendo otras variables como la formación, los recursos materiales, los apoyos personales con lo que cuenta el profesor y el centro educativo para conocer si indicen o no en las actitudes y estrategias de los docentes ya que son algunos de los aspectos que más han demandado los docentes.
Por último y no menos importante sigue siendo necesario realizar un trabajo longitudinal que involucre al equipo directivo y a las familias, es fundamental conocer la opinión y actitud de todos los implicados en el proceso educativo y evaluar si las actitudes de los profesionales hacia la inclusión de alumnos con discapacidad varían en función del tipo de discapacidad de cada uno y dificultan el derecho a una educación inclusiva. 
Referencias bibliográficas

Abellán, R. M. et al. (2010): "Una aproximación a la educación inclusiva en España”. Revista de educación inclusiva, 3 (I): I49-I64.

Abós, P., y Polaino, A. (1986): “Integración de deficientes mentales educables: un estudio de actitudes docentes”. Revista Española de Pedagogía, 44 (I72): I93-208.

Agran, M. et al. (2002): "Access to the general curriculum for students with significant disabilities: What it means to teachers". Education and Training in Mental Retardation and Developmental Disabilities, 37 (2): I23I33.

Ainscow, M. (20I 2): "Haciendo que las escuelas sean más inclusivas: lecciones a partir del análisis de la investigación internacional”. Revista de Educacióninclusiva, 5 (I): 39-49.

Ainscow, M. y Booth, T. (2000): Index for inclusion: Developing Learning and Participation in Schools. Developing Learning and Pin Schools, Bristol: CSIE.

Ainscow, M. et al. (2006): Improving schools, developing inclusion, Londres: Routledge.

Alemany, I. y Villuendas, M. D. (2004): “Las actitudes del profesorado hacia el alumnado con necesidades educativas especiales". Convergencia, (34): I83-215.

Álvarez, E. et al. (2008): “Funcionamiento de la integración en la Enseñanza Secundaria Obligatoria según la percepción del profesorado". Psicothema, 20 (I): 56-6I.

Arnaiz, P. (2004): "La educación inclusiva: dilemas y desafíos”. Educación, desarrollo y diversidad, $7(2): 25-40$.

Arnáiz, P. (I996): "Las Escuelas son para Todos”. Siglo cero, 27 (2): 25-34.

Avramidis, E., y Norwich, B. (2002): “Teachers’ attitudes towards integration/inclusion: A review of the literature". European Journal of Special Needs Education, I7 (2): I29-I47.
Avramidis, E. et al. (2000): “A survey into mainstream teachers' attitudes towards the inclusion of children with special educational needs in the ordinary school in one local education authority". Educational psychology, 20 (2): I9I-2II.

Ballone, L. M. y Czerniak, C. M. (200I): "Teachers' Beliefs about Accommodating Students' Learning Styles in Science Classes". Electronic Journal of Science Education, 6 (2): I-44.

Barrio de la Puente, J. L. (2009): "Hacia una educación inclusiva para todos”. Revista Complutense de Educación, 20 (I): I3-3 I.

Bhatnagar, N. y Das, A. (2OI4): “Attitudes of secondary school teachers towards inclusive education in New Delhi, India". Journal of Research in Special Educational Needs, I4 (4): $255^{-263 .}$

Bradfield, R. H. et al. (I973): "The special child in the regular classroom". Exceptional children, $39(5): 384-390$.

Cardona, M. C. (2006): Diversidad y educación inclusiva: enfoques metodológicos y estrategias para una enseñanza colaborativa, Madrid: Editorial Alhambra.

Cardona, M. C. (Abril de 2004): Getting ready for inclusion: Is the stage set in Spain?. En Annual Meeting of the American Educational Research Association. Congreso llevado a cabo en San Diego, Estados Unidos.

Cardona, M. C. et al. (2003): Demandas formativas del profesorado en relación a las adaptaciones de la enseñanza. En XI Congreso Nacional de Modelos de Investigación Educativa. Congreso llevado a cabo en Granada.

Cardona, M. C. et al. (2000): Cuestionario de Percepciones del Profesor acerca de una Pedagogía Inclusiva, Alicante: Universidad de Alicante 
Cardona, M. C. et al. (2000): Teoría y práctica de la adaptación de la enseñanza, Alicante: Universidad de Alicante.

Chiner, E. (20II): Las percepciones y actitudes del profesorado hacia la inclusión del alumnado con necesidades educativas especiales como indicadores del uso de prácticas educativas inclusivas en el aula (tesis doctoral). Universidad de Alicante, Alicante.

Cook, B. G. y Cameron, D. L. (2010): “Inclusive teachers' concern and rejection toward their students: Investigating the validity of ratings and comparing student groups". Remedial and Special Education, 3 I (2): 67-76.

Cook, L. y Friend, M. (I995): “Co-teaching: Guidelines for creating effective practices". Focus on Exceptional Children, 28 (3): I-I6.

Cook, B. G. et al. (2007): “Inclusive teachers' attitudinal ratings of their students with disabilities".The Journal of Special Education, $40(4): 230-238$.

Cornoldi, C. et al. (1998): "Teacher attitudes in Italy after twenty years of inclusion". Remedial and Special Education, I9 (6): 350-356.

Dart, G. (2007): "Provision for Learners with Special Educational Needs in Botswana: A Situational Analysis". International Journal of Special Education, 22 (2): 56-66.

De Boer, A. et al. (2012): “The psychometric evaluation of a questionnaire to measure attitudes towards inclusive education". European Journal of Psychology of Education, 27 (4): 573-589.

De Boer, A. et al. (20I I): "Regular primary schoolteachers' attitudes towards inclusive education: A review of the literature". International Journal of Inclusive Education, I $5(3): 33$ I-353.

Dendra, M. R. et al. (I99I): "Estudio de las variables que afectan a las actitudes de los maestros hacia la integración escolar de niños con necesidades especiales”. Anuario español e iberoamericano de investigación en educación especial, 2: 47-48.

Echeita, G. y Ainscow, M. (20I I): "La educación inclusiva como derecho. Marco de referencia y pautas de acción para el desarrollo de una revolución pendiente”. Tejuelo, (I 2): 26-46.

Escudero, J. M. (20I2): "La educación inclusiva, una cuestión de derecho”. Educatio Siglo XXI, 30 (2): IO9-I 28.

Flores, R. (2007): "Representaciones de género de profesores y profesoras de matemática, y su incidencia en los resultados académicos de alumnos y alumnas". Revista iberoamericana de educación, (43): I03-I I 8.

García, J. N. y Alonso, J. C. (I985): Actitudes de los maestros hacia la integración escolar de niños con necesidades especiales. Infancia y aprendizaje, 8 (30): 5I-68.

Granada, M. et al. (2013): “Actitud de los profesores hacia la inclusión educativa". Papeles de trabajo-Centro de Estudios Interdisciplinarios en Etnolingüística y Antropología Socio-Cultural, (25): 5 I-59.

Harasymiw, S. J. y Horne, M. D. (I975): "Integration of handicapped children: Its effect on teacher attitudes”. Education, 96 (2): I 53I 58 .

Higgs, R. W. (I975): “Attitude formation-contact or information”. Exceptional children, 4I (7): 496-497.

Horne, P. E. y Timmons, V. (2009): "Making it work: Teachers' perspectives on inclusion". International Journal of Inclusive Education, I3 (3): 273-286.

Huang, H. H. y Diamond, K. E. (2009): "Early childhood teachers' ideas about including children with disabilities in programmes designed for typically developing children". International Journal of Disability, Development and Education, 56 (2): I69-182.

Larrivee, B. y Cook, L. (I979): “Mainstreaming: A study of the variables affecting teacher attitude". The Journal of Special Education, I3: 3 I 5-324.

Leyser, Y. y Tappendorf, K. (200I): “Are attitudes and practices regarding mainstreaming changing? A case of teachers in two rural school districts”. Education, I2I (4): 75 I. 
Lou, Y. et al. (1996): "Within-class grouping: A meta-analysis". Review of educational research, $66(4): 423-458$.

Martínez Martín, M. A. y Bilbao León, M. C. (20II): "Los docentes de la universidad de Burgos y su actitud hacia las personas con discapacidad". Siglo Cero: Revista Española sobre Discapacidad Intelectual, 42 (240): $50-78$.

McCauley, R. W. et al. (I978): “The placement of handicapped children by Canadian public school personnel". Education and Training of the Mentally Retarded, I3 (4): 367-379.

McIntosh, R. et al. (I994): “Observations of students with learning disabilities in general education classrooms". ExceptionalChildren, $60(3): 249-26 I$.

Miroševi , J. K. y Lozan i , A. J. (20I4): “Attitudes of educators and teachers regarding the implementation of inclusion in regular preschools and primary schools. Hrvatska Revija Za Rehabilitacijska Istrazivanja, 50 (2): I7-29.

Nicasio, J. y Alonso, J. C. (I985): “Actitudes de los maestros hacia la integración escolar de niños con necesidades especiales”. Infancia $y$ aprendizaje, 8 (30): 5I-68.

Ovejero, A. (1998): Las Relaciones Humanas: Psicología Social Teórica y Aplicada, Madrid: Biblioteca Nueva.

Padeliadu, S. y Lampropoulou, V. (I997): "Attitudes of special and regular education teachers towards school integration". EuropeanJournal of Special Needs Education, I 2 (3): I73-I 83 .

Parrilla, A. (2002): "Acerca del origen y sentido de la educación inclusiva". Revista de educación, (327): I I-29.

Pernell, E. et al. (I985): "Mainstreaming: A continuing concern for teachers". Education, I06 (2): I3I-I37.

Sánchez, A. et al. (2008): "Percepciones y actitudes de los estudiantes de pedagogía hacia la inclusión educativa". Estudios pedagógicos (Valdivia), 34 (2): I69-I78.
Savage, L. B. y Wienke, W. D. (1989): “Attitude of secondary teachers toward mainstreaming”. The High School Journal, 73 (I): 70-73.

Scruggs, T. E. y Mastropieri, M. A. (2003): Inclusive classroom: Strategies for effective instruction. New Jersey: Prentice Hall.

Scruggs, T. E. y Mastropieri, M. A. (1996): "Teacher perceptions of mainstreaming/ inclusion, I958-I995: A research synthesis". Exceptional children, 63 (I): 59-74.

Stauble, K. R. (2009): Teacher attitudes toward inclusion and the impact of teacher and school variables (tesis doctoral). Universidad de Louisville, Estados Unidos.

Suriá, R. (20I2): "Discapacidad e integración educativa: ¿Qué opina el profesorado sobre la inclusión de estudiantes con discapacidad en sus clases?”. REOP-Revista Española de Orientación y Psicopedagogía, 23 (3): 96-109.

Triandis, H. C. (I97I): Attitude and attitude change, Nueva York: Wiley.

Unesco (I994): Declaración de Salamanca y Marco de acción ante las necesidades educativas especiales, París: Unesco.

Van Reusen, A. K. et al. (2000): "High school teacher attitudes toward inclusion". The High School Journal, 84 (2): 7-20.

Vaz, S. et al. (2015): "Factors associated with primary school teachers' attitudes towards the inclusion of students with disabilities". PLOS ONE, Iо (8): eOI37002.

Verdugo, M. A. (2009): "El cambio educativo desde una perspectiva de calidad de vida". Revista de Educación, 349 (2): 23-43.

Verdugo, M. A. (2003): "De la segregación a la inclusión escolar”, en Obra Social y Cultural Cajasur: Educar para la vida. Córdoba: Obra Social y Cultural Cajasur.

Verdugo, M. A. et al. (I995): “Actitudes sociales y profesionales hacia las personas con discapacidad: estrategias de evaluación e intervención”, en Verdugo, M. A. (ed.): Personas con discapacidad: Perspectivas psicopedagógicas y rehabilitadoras. Madrid: Siglo XXI de España Editores, S.A. 
Verdugo, M. A. et al. (I994): Actitudes hacia las personas con minusvalía. Madrid: Ministerio de Asuntos Sociales, Instituto Nacional de Servicios Sociales.

Vicuña, K. D. (2013): Percepciones y actitudes de profesores de primero a séptimo año de Educación General Básica del Distrito Educativo 4 hacia la inclusión del alumnado con necesidades educativas especiales como indicadores de uno de prácticas educativas inclusivas en el aula (tesis de maestría). Universidad Casa Grande, Ecuador. 